\title{
Avaliação dos resultados a longo prazo da cirurgia de Oberlin na paralisia braquial obstétrica*
}

\section{Evaluation of Long-Term Results of Oberlin Surgery in Obstetric Brachial Paralysis}

\author{
Eduardo Araújo Figueiredo ${ }^{10}$ Fernando Sellitti Chiabai de Freitas ${ }^{2}{ }^{\circledR}$ Júlio Inácio Parente Neto ${ }^{2}$ \\ Yussef Ali Abdouni ${ }^{3 \oplus}$ Antônio Carlos da Costa1
}

1 Departamento de Cirurgia da Mão, Santa Casa da Misericórdia de

São Paulo, Faculdade de Ciências Médicas, São Paulo, SP, Brasil

Endereço para correspondência Eduardo Araújo Figueiredo, Médico

2 Departamento de Ortopedia e traumatologia, Santa Casa da

Ortopedista especialista em Cirurgia de Mão e Microcirurgia, Rua Marquês de Itu, 836, apto 102, Consolação, São Paulo, SP, Brasil

Misericórdia de São Paulo, Faculdade de Ciências Médicas, São Paulo, SP, Brasil

${ }^{3}$ Departamento de Cirurgia da Mão e Microcirurgia, Faculdade de

Medicina, Universidade de São Paulo, São Paulo, SP, Brasil

(e-mail: figueiredo.eduardo@hotmail.com).

Rev Bras Ortop 2022;57(1):103-107.

\section{Resumo}

Palavras-chave

- plexo braquial

- paralisia obstétrica

- neuropatias do plexo braquial.
Objetivo Avaliar a flexão do cotovelo em crianças portadoras de paralisia obstétrica do plexo braquial submetidas à transferência de Oberlin.

Métodos Estudo retrospectivo com 11 pacientes acometidos por paralisia decorrente do trabalho de parto e que não apresentaram recuperação espontânea da flexão do cotovelo até os 12 meses de vida, operados entre 2010 e 2018.

Resultados As crianças foram operadas entre os 5 e 12 meses de vida, com média de 7,9 meses e o tempo médio de seguimento foi de 133,2 meses, variando de 37 a 238 meses. Seis pacientes $(54,5 \%)$ apresentaram grau de força muscular $\geq 3$, medido pela escala de força do Medical Research Council (MRC), e, pela escala de movimentação ativa (Active Momement Scale [AMS]), 5 pacientes obtiveram pontuação $\geq 5$. Foi identificada correlação negativa entre a AMS e a classificação de Narakas ( $r=-0,509)$, bem como entre a MRC e a classificação de Narakas $(r=-0,495)$. Já entre a AMS e a MRC, foi observada forte correlação positiva $(r=0,935)$, demonstrando que quanto maior a pontuação na escala de movimento, maior será a pontuação na escala de força muscular.

Conclusão A cirurgia de Oberlin apresenta-se como uma opção possível para a recuperação da flexão do cotovelo em crianças com plexopatia neonatal; no entanto, demonstra resultados bastante heterogêneos, mesmo no seguimento a longo prazo.

Trabalho desenvolvido na Santa Casa da Misericórdia de São Paulo, Faculdade de Ciências Médicas, São Paulo, Brasil.

recebido

26 de Abril de 2020

aceito após revisão

11 de Fevereiro de 2021

Publicado on-line

de Janeiro 21, 2022
DOI https://doi.org/

10.1055/s-0041-1731416.

ISSN 0102-3616. (c) 2022. Sociedade Brasileira de Ortopedia e Traumatologia. All rights reserved.

This is an open access article published by Thieme under the terms of the Creative Commons Attribution-NonDerivative-NonCommercial-License, permitting copying and reproduction so long as the original work is given appropriate credit. Contents may not be used for commercial purposes, or adapted, remixed, transformed or built upon. (https://creativecommons.org/ licenses/by-nc-nd/4.0/)

Thieme Revinter Publicações Ltda., Rua do Matoso 170, Rio de Janeiro, RJ, CEP 20270-135, Brazil 


\begin{abstract}
Objective To evaluate elbow flexion in children with obstetric brachial plexus paralysis submitted to Oberlin transfer.

Methods Retrospective study with 11 patients affected by paralysis due to labor who did not present spontaneous recovery from elbow flexion until 12 months of life, operated between 2010 and 2018.

Results The children were operated between 5 and 12 months of life, with a mean of 7.9 months, and the mean follow-up time was 133.2 months, ranging from 37 to 238 months. Six patients (54.5\%) presented a degree of muscle strength $\geq 3$, measured by the strength scale of the Medical Research Council (MRC) and, according to the active movement scale (AMS), 5 patients obtained a score of $\geq 5$. A negative correlation was identified between the AMS and the Narakas classification $(r=-0.509)$, as well as between the strength scale (MRC) and the Narakas classification $(r=-0.495)$. A strong positive correlation was observed $(r=0.935)$ between the AMS and the MRC demon-

\section{Keywords}

- brachial plexus

- paralysis, obstetric

- brachial plexus neuropathies strating that the higher the score on the movement scale, the higher the score on the muscle strength scale.

Conclusion The Oberlin surgery is a possible option for recovery of elbow flexion in children with neonatal plexopathy, demonstrating, however, very heterogeneous results, even in the long-term follow-up.
\end{abstract}

\section{Introdução}

As lesões do plexo braquial apresentam elevado potencial incapacitante, quando não tratadas de forma adequada. Em recém-nascidos, ocorrem no período expulsivo do parto, sendo muitas vezes associadas à distócia de ombro. Se porventura for aplicada uma força excessiva na manobra de extração, o ângulo entre o pescoço e o ombro é forçadamente aberto, causando um estiramento do plexo braquial. ${ }^{1}$ 0 resultado dessa tração pode variar desde neuropraxia até a avulsão das raízes nervosas. ${ }^{2,3}$

A primeira descrição clínica foi feita por Smellie em 1764. No entanto, o termo paralisia obstétrica (PO) foi atribuído pela primeira vez a Duchenne no ano de 1872, que descreveu com detalhes o quadro clínico de 4 pacientes. ${ }^{4,5} \mathrm{Em} 1874$, Erb estudou pacientes com lesões do tronco superior do plexo braquial e correlacionou seus dados com a descrição de Duchenne.

Em 1987, Narakas classificou as crianças com PO em 4 tipos: tipo 1, com lesão apenas das raízes de C5 e C6 (Erb); tipo 2, raízes de C5, C6 e C7 (Erb estendida) acometidas; tipo 3 , com lesão de todas as raízes do plexo; e tipo 4, que tem o sinal de Claude Bernard-Horner associado à lesão total. ${ }^{6}$

As lesões obstétricas do plexo braquial acometem com maior frequência as raízes de C5 e C6 (tipo 1 de Narakas) ou C5, C6, C7 (tipo 2). ${ }^{7}$ Nesses casos, o tratamento cirúrgico tem como um de seus principais objetivos o reestabelecimento da flexão do cotovelo, quando não ocorre a recuperação espontânea. O tempo para a realização da cirurgia, no entanto, permanece controverso, com autores indicando o limite entre os 6 e 9 meses de idade. ${ }^{6}$

No ano de 1994, Oberlin descreveu pela primeira vez a técnica de transferência fascicular do nervo ulnar para o ramo motor do nervo musculocutâneo que inerva o bíceps, sendo uma das técnicas preferidas dos cirurgiões na reconstrução do plexo braquial em adultos. ${ }^{8,9}$ As vantagens dessa técnica são o uso de um nervo doador previamente sadio e a realização de uma sutura nervosa em uma região sem fibrose e mais próxima ao músculo a ser reinervado, o que gera resultados superiores à reconstrução clássica com enxertos. ${ }^{10-15}$

Na literatura, a indicação deste tipo de neurotização em casos de PO tem sido pouco relatada em contraste com as lesões traumáticas no adulto, nos quais as indicações estão bem estabelecidas e já foram bastante descritas. ${ }^{8}$

O objetivo deste estudo é avaliar o resultado funcional a longo prazo, notadamente a flexão ativa do cotovelo em crianças portadoras de plexopatia neonatal submetidas à transferência de Oberlin.

\section{Casuística e métodos}

Foi realizado estudo retrospectivo com crianças portadoras de PO acompanhadas no ambulatório do grupo de mão e microcirurgia de hospital referência, no período entre janeiro de 2010 e dezembro de 2018. Foram incluídas do estudo as crianças que não apresentaram recuperação espontânea da flexão do cotovelo até os 12 meses de idade ou com força muscular menor que M2. Os critérios de exclusão foram: crianças maiores de um ano de idade; crianças antes submetidas a algum procedimento cirúrgico prévio no plexo braquial; crianças com lesão completa do plexo braquial que não recuperaram a função da mão; lesões bilaterais e paralisia cerebral associada.

Foram selecionados 11 pacientes que foram submetidos à cirurgia pela técnica de Oberlin, além da neurotização do 
nervo acessório para o nervo supraescapular. A dissecção intraneural, para identificação do fascículo motor para o músculo flexor ulnar do carpo, foi feita com o uso do microscópio, uma vez que o calibre destes nervos é muito menor em crianças pequenas. A sutura nervosa foi realizada sempre com fio mononylon 9-0.

Os dados coletados contemplaram: idade, o tempo entre a cirurgia e a avaliação, medidas de flexão do cotovelo pelas escalas de força (Medical Research Council [MRC]) e Active Movement Scale (AMS).

A análise estatística envolveu os dados descritivos, média, mediana, intervalo interquartil e desvio padrão, para as variáveis quantitativas: idade e tempo entre a cirurgia e avaliação final. As variáveis qualitativas foram expressas em frequências.

O teste de Kolmogorov-Smirnov (KS) foi utlizado para testar a normalidade das variáveis envolvidas. Sequencialmente, optou-se pela utilização da correlação de Spearman para verificar a correlação entre as medições de flexão de cotovelo (escalas MRC e AMS), classificação de Narakas, idade e tempo entre a cirurgia e avaliação final. Para a classificação do nível de correlação foi utilizado o seguinte sistema: $r \geq 0,9$ como correlação muito forte; $0,7 \geq r \leq 0,89$ como correlação forte; $0,5 \geq r \leq 0,69$ como correlação moderada; $0,3 \geq r$ $\leq 0,49$ como correlação fraca; $0 \geq r \leq 0,29$ como correlação muito fraca. ${ }^{16}$ Foi utilizado para computação dos dados o software IBM SPSS Statistics for Windows, Versão 21.0 (IBM Corp., Armonk, NY, EUA) considerando o nível de significância de $5 \%$.

Este estudo não recebeu nenhum suporte financeiro de fontes públicas, comerciais ou fins lucrativos. Além disso, a utilização dos dados de seres humanos foi aprovada pelo Comitê Nacional de Ética em Pesquisa (CAAE 25532619.0.0000.5479)

\section{Resultados}

A idade das crianças à realização da cirurgia variou de 5 a 12 meses, com média de 7,9 meses. Já o tempo médio decorrido entre a cirurgia e a avaliação foi de 133,2 meses, variando de 37 a 238 meses (-Tabela $\mathbf{1}$ ).

Com relação à classificação da lesão, 2 crianças eram do tipo 1 de Narakas, 5 do tipo 2 e 4 do tipo 3, com recuperação espontânea das raízes inferiores.

Considerando a escala MRC, 2 pacientes apresentaram M1, 3 M2, 3 M3 e 3 M4. Quanto à AMS, 2 apresentaram grau 1, 3 grau 3, um grau 4, 2 grau 5 e 3 grau 6 (-Tabela 2).

No que tange à correlação das variáveis analisadas, foi identificada correlação moderada e negativa entre a escala AMS e a classificação de Narakas $(r=-0,509)$, bem como, entre a escala MRC e a classificação de Narakas $(r=-0,495)$, sendo essa última considerada fraca. Assim, quanto maior a pontuação nas escalas apresentadas, menor será a graduação da lesão na classificação de Narakas. Houve correlação fraca e positiva entre a escala AMS e a idade do paciente quando da cirurgia $(r=0,364)$ e o tempo entre a cirurgia e a avaliação $(r=0,343)$, bem como a escala MRC e a idade $(r=0,411)$ e $o$ tempo de evolução $(r=0,301)$. Ou seja, quanto maior for a
Tabela 1 Descritiva completa para variáveis Quantitativas idade quando da cirurgia e tempo de evolução

\begin{tabular}{|l|l|l|}
\hline Descritiva & $\begin{array}{l}\text { Idade na } \\
\text { cirurgia } \\
\text { (meses) }\end{array}$ & $\begin{array}{l}\text { Tempo de } \\
\text { evolução } \\
\text { (meses) }\end{array}$ \\
\hline Média & 7,9 & 133,2 \\
\hline Mediana & 6 & 160 \\
\hline Desvio padrão & 2,7 & 63,4 \\
\hline Coeficiente de variação & $35 \%$ & $48 \%$ \\
\hline Mínimo & 5 & 37,08 \\
\hline Máximo & 12 & 237,6 \\
\hline Número de indivíduos & 11 & 11 \\
\hline Intervalo de confiança & 1,6 & 37,5 \\
\hline
\end{tabular}

idade das crianças no momento da cirurgia ou o tempo de evolução entre o ato cirúrgico e a avaliação final, maior será a pontuação nas escalas apresentadas. Quando envolvemos a correlação entre as escalas AMS e e MRC, foi identificada correlação muito forte e positiva $(r=0,935)$, demonstrando que quanto maior a pontuação na escala de movimento, maior será a pontuação na escala de força muscular (-Tabela 3).

\section{Discussão}

As lesões do plexo braquial no nascimento acometem com maior frequência o tronco superior; ${ }^{17}$ assim, a flexão do cotovelo está invariavelmente comprometida. Apesar do alto índice de recuperação espontânea nos 6 primeiros meses de vida, nos casos em que isso não ocorre, a ausência da flexão do cotovelo é extremamente incapacitante para a função do membro superior. ${ }^{2,18}$ Nessas situações, o tratamento cirúrgico está indicado para a reconstrução primária do plexo braquial, podendo ser realizado até os 12 meses de idade, mesmo não havendo um consenso na literatura sobre a idade ideal. $4,11,19-21$

Apesar da cirurgia de Oberlin estar bem estabelecida para o tratamento das lesões em adultos, ela tem sido pouco

Tabela 2 Distribuição das variáveis qualitativas

\begin{tabular}{|l|l|l|l|}
\hline \multicolumn{2}{|c|}{} & $\begin{array}{l}\text { Número de } \\
\text { indivíduos }\end{array}$ & $\%$ \\
\hline $\begin{array}{l}\text { AMS - active } \\
\text { movement scale }\end{array}$ & AMS 1 & 2 & $18,2 \%$ \\
\cline { 2 - 4 } & AMS 3 & 3 & $27,3 \%$ \\
\cline { 2 - 4 } & AMS 4 & 1 & $9,1 \%$ \\
\cline { 2 - 4 } & AMS 5 & 2 & $18,2 \%$ \\
\cline { 2 - 4 } & AMS 6 & 3 & $27,3 \%$ \\
\hline \multirow{4}{*}{$\begin{array}{l}\text { MRC - Medical } \\
\text { Rearch Council }\end{array}$} & M1 & 2 & $18,2 \%$ \\
\cline { 2 - 4 } & M2 & 3 & $27,3 \%$ \\
\cline { 2 - 4 } & M3 & 3 & $27,3 \%$ \\
\cline { 2 - 4 } & M4 & 3 & $27,3 \%$ \\
\hline
\end{tabular}


Tabela 3 Correlação entre a escala de movimento de flexão do cotovelo e a classificação de Narakas, idade do paciente quando da cirurgia e tempo entre a cirurgia e a avaliação

\begin{tabular}{|l|l|l|l|l|l|}
\hline \multicolumn{2}{|c|}{} & Classificação de Narakas & Idade cirurgia & Tempo evolução & MRC \\
\hline \multirow{2}{*}{ MRC } & Correlação Spearman $(r)$ & $-0,495$ & 0,411 & 0,301 & \\
\cline { 2 - 6 } & Valor de $p$ & 0,121 & 0,209 & 0,369 & \\
\hline \multirow{2}{*}{ AMS } & Correlação Spearman $(r)$ & $-0,509$ & 0,364 & 0,343 & 0,935 \\
\cline { 2 - 6 } & Valor de $p$ & 0,109 & 0,271 & 0,302 & $<\mathbf{0 , 0 0 1}$ \\
\hline
\end{tabular}

Abreviaturas: AMS, active movement scale; MRC, Medical Research Council.

relatada na literatura em casos de PO. 4,11,19,22 Tal fato se deve à ocorrência de recuperação espontânea do bíceps na grande maioria dos casos de PO e, portanto, a indicação da cirurgia é menos frequente do que em adultos. Isso justifica também o fato de os trabalhos encontrados na literatura não apresentarem grandes casuísticas, variando de 7 a 20 casos, ${ }^{20}$ compatíveis com o presente estudo.

Por realizar a sutura nervosa mais próxima ao músculo a ser reinervado, essa técnica permite a realização de cirurgias primárias em crianças até os 2 anos de vida com resultados funcionais, como observado por outros autores. ${ }^{24}$ Decorrente a esse fato, o método em estudo torna viável o tratamento de crianças que apenas se apresentam a um serviço especializado com mais de um ano de vida. Semelhante aos demais estudos na literatura, não foi observada no presente estudo qualquer disfunção, sensitiva ou motora, na mão dos pacientes decorrente da transferência fascicular do nervo ulnar.

Um dos desafios na avaliação dos resultados é instituir parâmetros objetivos para avaliar a flexão ativa do cotovelo que sejam reprodutíveis entre diferentes observadores. A escala de AMS foi desenvolvida para avaliar crianças menores com PO, sendo usada por diversos autores. ${ }^{2,24}$ No entanto, a escala MRC, mesmo não sendo tão específica, é mais difundida e preferida por outros autores. ${ }^{15,23}$ Apesar das diferenças na graduação entre as escalas, observamos correspondência entre as duas no resultado a longo prazo da força muscular de flexão do cotovelo.

Os resultados deste estudo, porém, não foram homogêneos e destoaram da maior parte da literatura, que aponta bons resultados para a flexão do bíceps com a cirurgia de Oberlin na PO. Little el al. observaram, em um estudo retrospectivo de 31 casos, $77 \%$ de recuperação da flexão do cotovelo contra a gravidade. ${ }^{12}$

Seis de 11 crianças (54,5\%) apresentaram, na escala MRC, força contra gravidade ou maior (3 crianças grau 3 e 3 crianças grau 4) e 5 apresentaram, na escala AMS, movimentação ativa contra gravidade ou maior (2 crianças grau 5 e 3 crianças grau 6). De acordo com Clarke e Curtis (1995), são consideradas funcionais as amplitudes de movimento que ultrapassem $50 \%$ contra a gravidade, ou seja, AMS $=6 .{ }^{25}$

Os resultados divergentes desta pesquisa podem ser creditados à ativação simultânea do tríceps quando o paciente tenta fletir o cotovelo. Esse fenômeno se tornou evidente quando, apesar da contração visível e palpável do bíceps, a ação antagônica do tríceps impediu o movimento em alguns casos. Alguns autores têm descrito os resultados da aplicação de toxina botulínica no músculo tríceps para tratar essa condição e melhorar a flexão ativa do cotovelo. ${ }^{2}$ No entanto, ainda não há estudos sobre o uso da toxina botulínica para tratar a co-contração entre o músculo tríceps e o bíceps na reabilitação da cirurgia de Oberlin.

Este estudo destaca-se pelo longo tempo de seguimento, que foi maior que o observado nos trabalhos publicados até $o$ presente momento. Siqueira et al., ${ }^{26}$ publicaram o seguimento de 8 pacientes após um tempo médio de 8,3 anos, entretanto, o objetivo fora avaliar a função da mão.

Teoricamente, os resultados para flexão do cotovelo tendem a se mostrar superiores quanto maior for o tempo de evolução. ${ }^{14}$ Contudo, não foram observadas diferenças estatisticamente significantes, quando analisados os resultados com o tempo de evolução. Da mesma forma, não houve relação com a idade da realização da cirurgia, como havia observado também o estudo de Murison. ${ }^{24}$ Uma casuística maior poderia acrescentar mais evidência a estas observações. Ainda assim, como se trata de uma indicação pouco frequente, estudos multicêntricos reunindo experiências de vários grupos especializados poderiam ser realizados para atingir esse propósito.

\section{Conclusão}

A cirurgia de Oberlin apresenta-se como uma opção possível para a recuperação da flexão do cotovelo em crianças com plexopatia neonatal; no entanto, demonstra resultados bastante heterogêneos, mesmo no seguimento a longo prazo.

\section{Suporte Financeiro}

Não houve suporte financeiro de fontes públicas, comerciais, ou sem fins lucrativos.

Conflito de Interesses

Os autores declaram não haver conflito de interesses.

\section{Referências}

1 Chang KWC, Wilson TJ, Popadich M, Brown SH, Chung KC, Yang LJS. Oberlin transfer compared with nerve grafting for improving early supination in neonatal brachial plexus palsy. J Neurosurg Pediatr 2018;21(02):178-184

2 Figueiredo RdeM, Grechi G, Gepp RdeA. Oberlin's procedure in children with obstetric brachial plexus palsy. Childs Nerv Syst 2016;32(06):1085-1091 
3 Gilbert A, Brockman R, Carlioz H. Surgical treatment of brachial plexus birth palsy. Clin Orthop Relat Res 1991;(264):39-47

4 Gilbert A, Pivato G. Obstetrical palsy: The french contribution. Semin Plast Surg 2005;19(01):5-16

5 Terzis JK, Kokkalis ZT. Elbow flexion after primary reconstruction in obstetric brachial plexus palsy. J Hand Surg Eur Vol 2009;34 (04):449-458

6 Narakas AO. Obstetrical Brachial Plexus Injuries. In: Lamb DW, ed. The paralysed hand. Edimburgh: Churchill Livingstone; 1987: 116-135

7 Rodrigues DB, Viegas MLC, Rogério JS, Pereira ELR. Tratamento cirúrgico das lesões traumáticas do plexo braquial. Arq Bras Neurocir 2014;33(02):125-131

8 Rezende MR, Rabelo NTA, Silveira CCJ, Petersen PA, De Paula EJL, Mattar RJ. Resultado da neurotização do nervo ulnar para o músculo bíceps braquial na lesão do plexo braquial. Acta Ortop Bras 2012;20(06):317-323

9 Corrêa MG, Cardoso MM, Gepp RA, Quiroga MRS, Beraldo PSS. Brachial plexus injuries with ulnar musculocutaneous transfer: 78 cases study focused on possible postoperative complications. Arq Bras Neurocir 2019;38(01):7-11

10 Al-Qattan MM, Thallaj A, Abdelhamid MM. Ulnar nerve to musculocutaneous nerve transfer in an ulnar ray-deficient infant with brachial plexus birth palsy: case report. J Hand Surg Am 2010;35 (09):1432-1434

11 Leechavengvongs S, Witoonchart K, Uerpairojkit C, Thuvasethakul P, Ketmalasiri W. Nerve transfer to biceps muscle using a part of the ulnar nerve in brachial plexus injury (upper arm type): a report of 32 cases. J Hand Surg Am 1998;23(04):711-716

12 Little KJ, Zlotolow DA, Soldado F, Cornwall R, Kozin SH. Early functional recovery of elbow flexion and supination following median and/or ulnar nerve fascicle transfer in upper neonatal brachial plexus palsy. J Bone Joint Surg Am 2014;96(03):215-221

13 Oberlin C, Béal D, Leechavengvongs S, Salon A, Dauge MC, Sarcy JJ. Nerve transfer to biceps muscle using a part of ulnar nerve for $\mathrm{C} 5$ C6 avulsion of the brachial plexus: anatomical study and report of four cases. J Hand Surg Am 1994;19(02):232-237

14 Shigematsu K, Yajima H, Kobata Y, Kawamura K, Maegawa N, Takakura Y. Oberlin partial ulnar nerve transfer for restoration in obstetric brachial plexus palsy of a newborn: case report. Journal of Brachial Plexus and Peripheral Nerve Injury 2006;1(01):e39-e43
15 Siqueira MG, Socolovsky M, Heise CO, Martins RS, Di Masi G. Efficacy and safety of Oberlin's procedure in the treatment of brachial plexus birth palsy. Neurosurgery 2012;71(06): 1156-1160

16 Hulley SB, Cummings SR, Browner WS, Grady DG, Newman TB. Delineando a pesquisa clínica: uma abordagem epidemiológica. 3a. ed. São Paulo: Artmed; 2008

17 Kozin SH. Nerve transfers in brachial plexus birth palsies: indications, techniques, and outcomes. Hand Clin 2008;24(04): 363-376

18 Arias AV, Gonçalves VMG, Campos D, Santos DCC, Goto MMF, Campos Zanelli TM. Desenvolvimento das habilidades motoras finas no primeiro ano de vida. Rev Neurocienc 2010;18(04): 544-554

19 Ghanghurde BA, Mehta R, Ladkat KM, Raut BB, Thatte MR. Distal transfers as a primary treatment in obstetric brachial plexus palsy: a series of 20 cases. J Hand Surg Eur Vol 2016;41(08): 875-881

20 Fisher DM, Borschel GH, Curtis CG, Clarke HM. Evaluation of elbow flexion as a predictor of outcome in obstetrical brachial plexus palsy. Plast Reconstr Surg 2007;120(06):1585-1590

21 Pondaag W, Malessy M. The Evidence for Nerve Repair in Obstetric Brachial Plexus Palsy Revisited. Biomed Res Int 2014;2014:1-11

22 Garg R, Merrell GA, Hillstrom HJ, Wolfe SW. Comparison of nerve transfers and nerve grafting for traumatic upper plexus palsy: a systematic review and analysis. J Bone Joint Surg Am 2011;93 (09):819-829

23 Noaman HH, Shiha AE, Bahm J. Oberlin's ulnar nerve transfer to the biceps motor nerve in obstetric brachial plexus palsy: indications, and good and bad results. Microsurgery 2004;24(03): 182-187

24 Murison J, Jehanno P, Fitoussi F. Nerve transfer to biceps to restore elbow flexion and supination in children with obstetrical brachial plexus palsy. J Child Orthop 2017;11(06):455-459

25 Clarke HM, Curtis CG. An approach to obstetrical brachial plexus injuries. Hand Clin 1995;11(04):563-580

26 Siqueira MG, Heise CO, Pessa M, Zacariotto M, Martins RS. Longterm evaluation of hand function in children undergoing Oberlin and Oberlin-like procedures for reinnervation of the biceps muscle. Childs Nerv Syst 2020;36(12):3071-3076 